\title{
Gold Screen-printed Electrodes as detectors of reducing sugars in batch and FIA systems
}

\author{
Pablo Fanjul Bolado ${ }^{1}$, Marta M.P.S. Neves ${ }^{1}$ María Begoña González García ${ }^{1}$, David Hernández \\ Santos ${ }^{1}$ \\ ${ }^{1}$ DropSens, S.L. Edificio CEEl, Parque Tecnológico de Asturias, 33428, Llanera, Asturias, Spain \\ Corresponding author: pfanjul@dropsens.com
}

\begin{abstract}
The development of user-friendly, time-saving and cost-effective analytical strategies for the detection and quality control of carbohydrates in food industry is continuously highly demanded. Therefore, in this work, two strategies for the non-enzymatic detection of reducing sugars (glucose and fructose), employing miniaturized screen-printed gold electrodes, were proposed.

On the one hand, a high-throughput flow-injection analysis (FIA) system that employed gold electrodes integrated in a one channel flow-cell was developed. The injection of sample volume (60 $\mu \mathrm{L}$ ) was done through an "in-line luer" injection port, placed closest to the electrochemical cell, and highly controlled by operator through a syringe. This configuration brings important advantages since it simplifies operability and effectiveness of working in FIA systems. The sugars detection was carried out by applying a potential of $+0.5 \mathrm{~V}$ in a $\mathrm{NaOH} 0.1 \mathrm{M}$ flow carrier stream of $1 \mathrm{ml} \mathrm{min}^{-1}$. On the other hand, a batch mode approach using disposable gold electrodes for sugars detection by dropping the sample $(60 \mu \mathrm{L})$ directly in the electrochemical cell was also investigated. The analytes were measured amperometrically, by applying a constant potential of $+0.5 \mathrm{~V}$ during $2 \mathrm{~min}$, and by determining the anodic oxidation peak current by cyclic voltammetry. Accuracy and precision studies were performed and the limits of detection and linear ranges were established. Finally, the applicability of the developed non-enzymatic sensors has been successfully evaluated by determining reducing sugars in commercial foodstuffs.
\end{abstract}

Key words: reducing sugars, screen-printed sensor, flow system, thin layer flow-cells, portable instruments

\section{Introduction}

Carbohydrates detection has assumed relevant importance in different areas, especially in the food and beverage industry. Among of them, molecules such as glucose, fructose, maltose and lactose are considered reducing sugars. For the detection of these kind of sugars, different and successful enzymatic sensors have been widely developed [1], which are characterized by high sensitivity, specificity and selectivity. However, these sensors show the known disadvantages related to the use of enzymes, such as the chemical and thermal instability. Therefore, as an alternative, different strategies with non-enzymatic sensors based on metals have been reported and exploited. Metals such as nickel, copper, platinum and gold are among the most widely studied [2]. Respect to the process of electrocatalysis of glucose, fructose, and other organic molecules, many studies indicate that it coincides with the appearance of adsorbed hydroxyl groups (OHads) on the metal surface, which participate in the oxidation of adsorbed species. Although there is not a consensus regarding these mechanisms in the literature, as a general rule, the OHads are pointed out as responsible for the electrocatalytic behavior of noble metals. In the case of gold electrodes, it is believed that the catalytic component, more pronounced at higher pHs, is the hydrated gold oxide, $\mathrm{AuOH}$, which is formed by the chemisorption of hydroxide anions on the surface of the gold.

In this work gold screen-printed electrodes were used as reducing sugar sensors. Two different configurations were tested, one of them for bath analysis and the other for flow injection analysis. The methodology was optimized in both cases. After, an application of these sensors in the determination of reducing sugars 
in honey samples was shown, obtaining good results.

\section{Experimental Section}

Batch detection. The methodology consisted on dropping on the electrochemical cell of a disposable gold screen printed electrodes (DRP-220AT) an aliquot of $60 \mathrm{uL}$ of sample diluted in $0.1 \mathrm{M} \mathrm{NaOH}$. Cyclic voltammetry scan was registered between $0.0 \mathrm{~V}$ and $+1.0 \mathrm{~V}$ with a scan rate of $50 \mathrm{mV} / \mathrm{s}$. The anodic peak current was measured for each sample.

FIA detection. In this case a gold electrode integrated in a one channel flow cell (DRPTLFCL210AT-CIR, figure 1A) was used as detector, The injection of $60 \mathrm{uL}$ of sample was done through a syringe and an in-line luer injection port, placed closest the electrochemical cell (Figure 1B). An amperometric detection was performed by appling a constant potential of $+0.5 \mathrm{~V}$ in a $\mathrm{NaOH} 0.1 \mathrm{M}$ flow carrier (1 mL/min).
A)

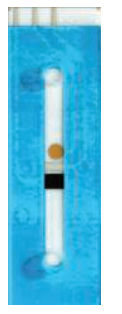

B)

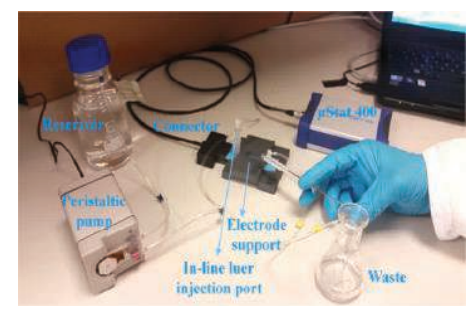

Fig. 1. A) Thin layer flow-cell gold screen-printed electrode, circular working electrode; B) FIA system

\section{Results}

Bath detection. Good calibration plots were obtained for concentrations of sugar (glucose + fructose) (figure $2 \mathrm{~A}$ ) comprised between 0.05 $\mathrm{mM}$ and $5.0 \mathrm{mM}$ with a good inter- and intrabatch reproducibility $(1<\mathrm{RSD}(\%)<15)$. The quantification of reducing sugars in honey samples was carried out with good accuracy (88-102 \%).

FIA detection. Good calibration plots were obtained for concentrations of sugar (glucose + fructose) comprised between $0.01 \mathrm{mM}$ and 1.0 $\mathrm{mM}$ (coefficient correlation of 0.997). The reproducibility of consecutive injections was around $6.5 \%$ (in terms of \%RSD) and no memory effect was observed (figure 2B). The quantification of reducing sugars in honey samples was performed with good accuracy $(91-113 \%)$ without any sample pretreatment.
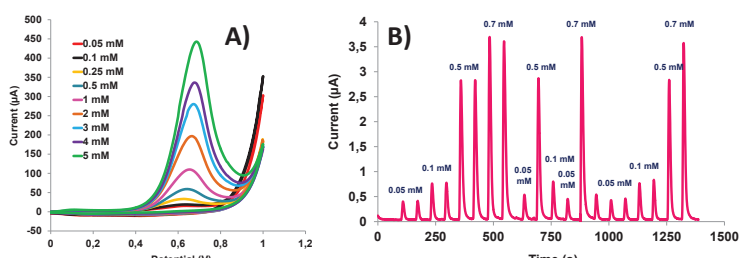

Fig. 2. A) Cyclic voltammograms obtained for reducing sugar concentrations comprised into the calibration plot; B) Consecutive injections of different concentrations of reducing sugars in FIA system

\section{Conclusions}

Two non-enzymatic methods based on gold screen-printed electrodes were optimized for detection of reducing sugar, achieving accuracy results when they were applied to real honey samples. The good results obtained with honey are being achieved with other kind of samples $i$ e. fermentation must and wine samples (preliminary results not shown in this work) and although both methodologies could be used interchangeably without any significant difference, it may be advisable to use the FIA system when a high number of samples needs to be analyzed whereas the bacth system could be used for field applications with the help of user-frendly and portable instruments (electrochemical readers).

\section{Acknowledgements}

This work was supported by the MANUNET ERA-NET Project "SENS4WINE" (IDE/2016/0000157), co-financed through IDEPA and ERDF fund.

\section{References}

[1] A. Heller, B. Feldman, Electrochemical Glucose Sensors and their Applications in Diabetes Management, Chem.Rev.108 (2008) 2482-2505; doi: 10.1021/cr068069y

[2] K.E. Toghill, R.G. Compton, Electrochemical Non-enzymatic Glucose Sensors: a Perspective and an Evaluation, Int. J. Electrochem. Sci., 5 (2010) 1246-1301. 